\title{
A Study on Dispersion Law of Underwater Threat based on Monte Carlo Algorithm
}

\author{
Shufen LIUa, Ji HOUb, Lu HANc,* \\ College of Computer Science and Technology \\ Jilin University \\ Changchun 130012, China \\ E-mail: aliusf@jlu.edu.cn \\ E-mail: ${ }^{\mathrm{b}} 1044390409 @ q q . c o m$ \\ *E-mail: ${ }^{c}$ hanlu@jlu.edu.cn \\ $+*$ Corresponding author
}

\begin{abstract}
At present, it is impossible to detect the underwater target accurately found at a distance by equipment such as multi-sonars due to the limitation of underwater target detection technology. A method using Monte Carlo algorithm to confirm the dispersion law of underwater threat according to the target motion elements is presented. Combined with the probability theory, probability density of underwater threat locating is calculated. Finally, the distribution law of the typical situation is simulated, and the simulation results show that the profile of probability density distribution of the threat is consistent with the result of the threat distribution law.
\end{abstract}

Keywords-monte carlo; analysis of underwater target distribution; probability density of underwater target position distribution

\section{INTRODUCTION}

The dispersion law of underwater threat plays an important role in the decision-making of commanders, and it is an important guidance basis for naval detection and operation. Previous studies have paid little attention to the distribution law of targets[1,2,3,4,6]. A large number of simplifying assumptions are given to get a rough target distribution probability density function $[1,2,3,5]$. Simplified distribution law of target area is convenient for real-time decision-making, but in some cases, the error caused by simplification may affect the effectiveness of decision-making.

Monte Carlo method is based on mathematical statistics, which is widely applied to many fields such as physics, mathematics, biology, machinery and economics [7, 8, 9]. Monte Carlo (MC for short) method is an effective means to comprehensively analyze the dispersion law of underwater threat and calculate the distribution density function. The statistical results can analyze and test the validity of the present underwater target distribution model in different situations, which can help the commanders to make effective and tactical decision, and improve the success rate of target searching and uncovering.

\section{MODELING}

\section{A. Description of Underwater Threat Dispersion}

Underwater threat dispersion refers to the distribution of underwater targets in a particular area at a given moment.
For the maneuvering of underwater target, generally speaking, the target motion can be divided into two cases: the known target motion element and the estimated target motion element. Among them, the known target movement factor is the most common situation [1].This article will only consider this kind of situation.

Target motion elements mainly include azimuth,distance, speed and heading targets .All of them obey normal distribution, and their standard deviations are marked as $\sigma a, \sigma r, \sigma v$ and $\sigma d$ respectively. The mean values are marked as A0, R0, V0 and D0.

\section{B. Modeling of Underwater Threat Dispersion}

The moment when a commander gains the target motion element is used as the initial moment, and the detection node location of the initial moment is used as the coordinate origin. A two plane rectangular Cartesian coordinate system is established whose upward direction represents the north, and downward direction represents the south, namely the forward direction of $\mathrm{x}$ axis represents the east direction on the map, the forward direction of $y$ axis represents the north,as is shown in Figure.1.

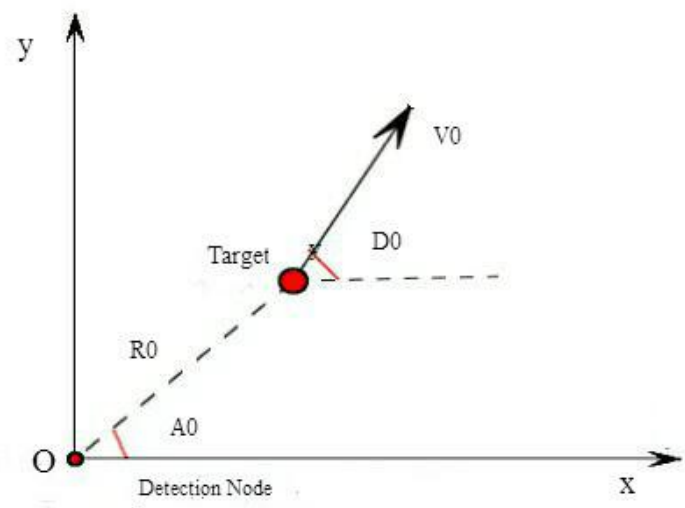

Figure 1. Schematic diagram of underwater threat dispersion

Known variables are the initial azimuth of target A0, distance of target R0, target velocity V0 and heading of target D0. Develop an algorithm to work out the position distribution of the target and the probability density function of the target position distribution and probability density map of the target position distribution at moment $t$. 


\section{PROBLEM SOLVING}

\section{A. Target Position Distribution Law at Moment t}

The target $(\mathrm{x}, \mathrm{y})$ position distribution law at moment $\mathrm{t}$ can be determined by azimuth (A), distance (R), velocity (V) and heading (D). The expression is as follows.

$$
\left\{\begin{array}{l}
x=D \cos F+V t \cos H \\
y=D \sin F+V t \sin H
\end{array}\right.
$$

Obviously they are fairly complex analytic functions, and it is difficult to predict the probability density function of the target distribution accurately. Therefore, the MC statistical simulation method is an effective means to comprehensively analyze the dispersion law of underwater threat and calculate the distribution density function $[8,9,10]$.

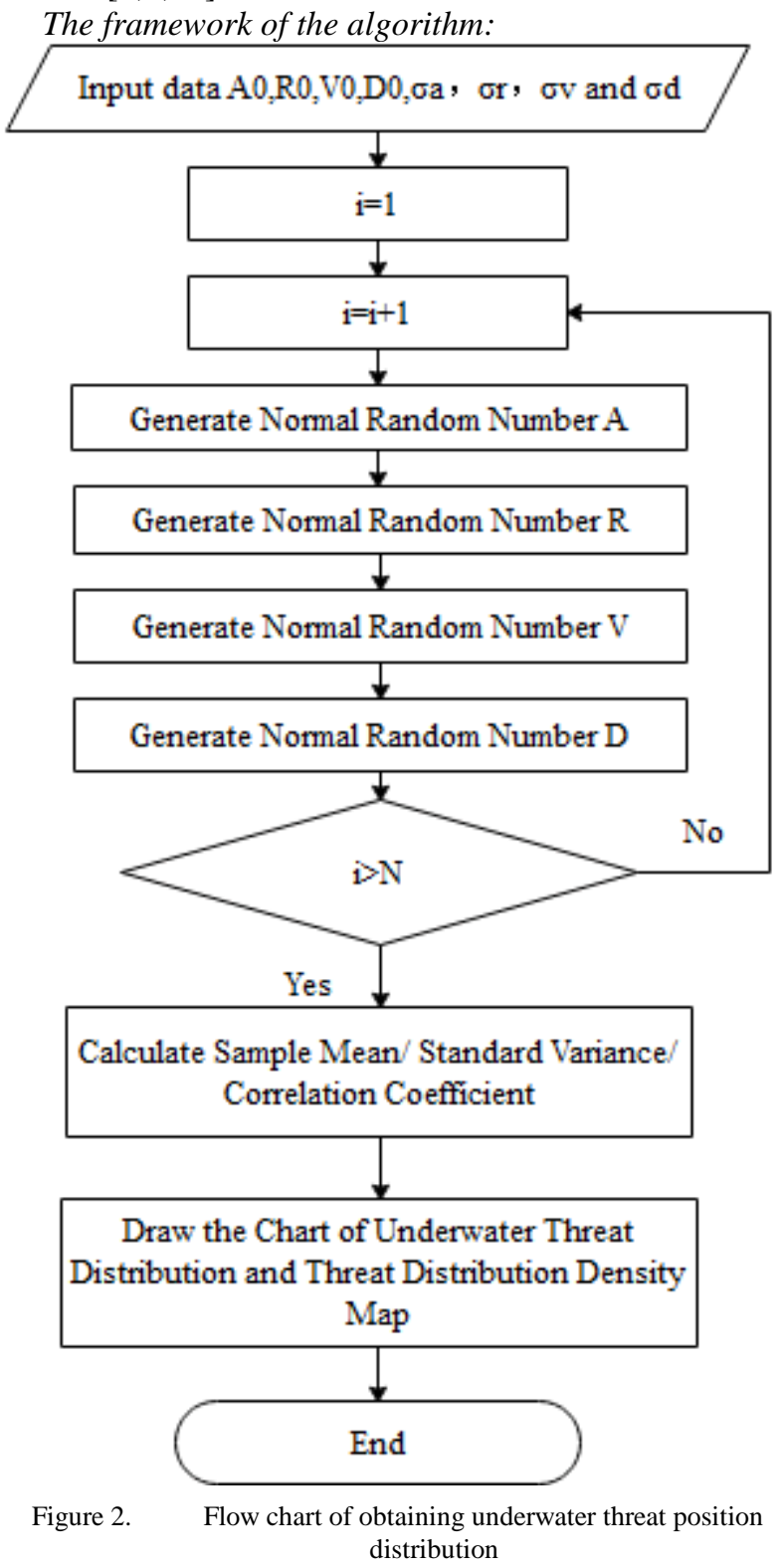

The steps to obtain the dispersion law of underwater threat are as follows.
(1)Assume that the azimuth of the target is A, and which obeys the normal distribution of $(\mathrm{A} 0, \sigma \mathrm{a})$. Then we generate the normal random number array $\mathrm{A}=(\mathrm{a} 1, \mathrm{a} 2, \mathrm{a} 3 \ldots \mathrm{an})$;

(2)Assume that the distance of the target is R, and which obeys the normal distribution of $(\mathrm{R} 0, \sigma \mathrm{r})$. Then we can generate the normal random number array $\mathrm{R}=(\mathrm{r} 1, \mathrm{r} 2$, r3...rn);

(3) Assume that the velocity of the target is V, and which obeys the normal distribution of $(\mathrm{V} 0, \sigma \mathrm{v})$. Then we generate the normal random number array $\mathrm{V}=(\mathrm{v} 1, \mathrm{v} 2, \mathrm{v} 3 \ldots \mathrm{vn})$;

(4) Assume that the heading of the target is D, and which obeys the normal distribution of (D0, $\sigma \mathrm{d})$. Then we generate the normal random number array $\mathrm{D}=(\mathrm{d} 1, \mathrm{~d} 2$, d3...dn);

(5) According to the formula (1), a corresponding two-dimensional array of random sample $(X 1, Y 1)=\{(x 1$, $\mathrm{y} 1),(\mathrm{x} 2, \mathrm{y} 2) \ldots(\mathrm{xn}, \mathrm{yn})\}$ is obtained;

(6) Repeat steps (1)-(5) for N/n times, we can generate $\mathrm{N}$ random samples $(\mathrm{Xi}, \mathrm{Yi})$. As is known to us all, each sample(xi, yi) can be represented by a point in a rectangular coordinate system. And so we can draw clearly the size and shape of the underwater threat distribution area if the number of samples could be large enough;

(7) Draw the image of the underwater threat dispersion according to the sample.

\section{B. Probability Density Function of Target Position Distribution at moment $t$}

The study found that the dispersion law of underwater threat obeys normal distribution. We can generate the probability density function in accordance with the knowledge of probability distributions and principles of statistics with a sufficient number of samples.

The probability density function of the target position distribution at moment $t$ is calculated as follows.

1) Calculate the sample mean of $x$

$$
U x=\frac{1}{N} \sum_{i=1}^{N} x_{i}
$$

2) Calculate the sample mean of $y$

$$
U y=\frac{1}{N} \sum_{i=1}^{N} y_{i}
$$

3) Calculate the standard deviation of $\mathrm{x}$

$$
\sigma_{x}=\sqrt{\frac{\sum_{i=1}^{n}\left(x_{i}-\boldsymbol{U}_{x}\right)^{2}}{N}}
$$

4) Calculate the standard deviation of $y$

$$
\sigma y=\sqrt{\frac{\sum_{i=1}^{n}\left(y_{i}-u_{y}\right)^{2}}{N}}
$$


5) Calculate the correlation coefficients of $x$ and $y$

$$
\rho=\frac{\operatorname{Cov}(X, Y)}{\sqrt{\sigma x \sigma y}}
$$

Then we can get the probability density function of the target position distribution at moment $t$ as following.

$$
f(x, y)=\frac{1}{2 \pi \sigma_{x} \sigma_{y} \sqrt{1-\rho^{2}}} e^{-\frac{1}{2\left(1-\rho^{2}\right)}\left(\frac{u_{x}^{2}}{\sigma_{x}^{2}}-\frac{2 \rho u_{x} u_{y}}{\sigma_{x} \sigma_{y}}+\frac{u_{y}^{2}}{\sigma_{y}^{2}}\right)}
$$

\section{EXAMPLE}

In order to verify the validity of this model, a method of calculation and simulation is used to describe the distribution of the target location for a typical tactical situation, and we conduct a comparative study.

Known:

$$
\sigma_{a}=1.4826 \sigma_{r}=2.9652 \sigma_{v}=0.593 \sigma_{d}=1.4826
$$

Assume that the target distance $\mathrm{R} 0=240 \mathrm{cab}$, target heading $\mathrm{A} 0=300$, target speed $\mathrm{V} 0=18 \mathrm{kn}$, and the target course $\mathrm{H} 0=90$ degree at the initial moment [2].

Draw the chart of underwater threat distribution using Python's Matplotlib library, as is shown in Figure.3.

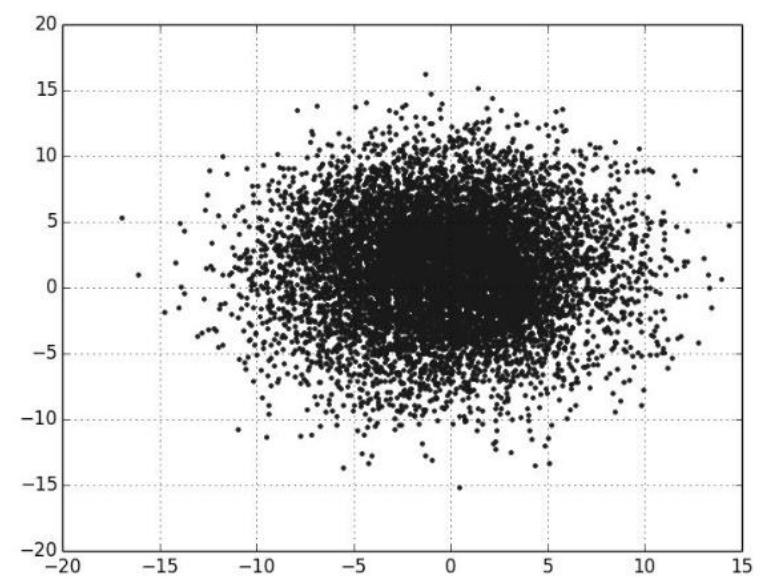

Figure 3. Schematic diagram of underwater threat dispersion

Draw the chart of probability distribution density of underwater threat using Python's Matplotlib library, as is shown in Figure.4.

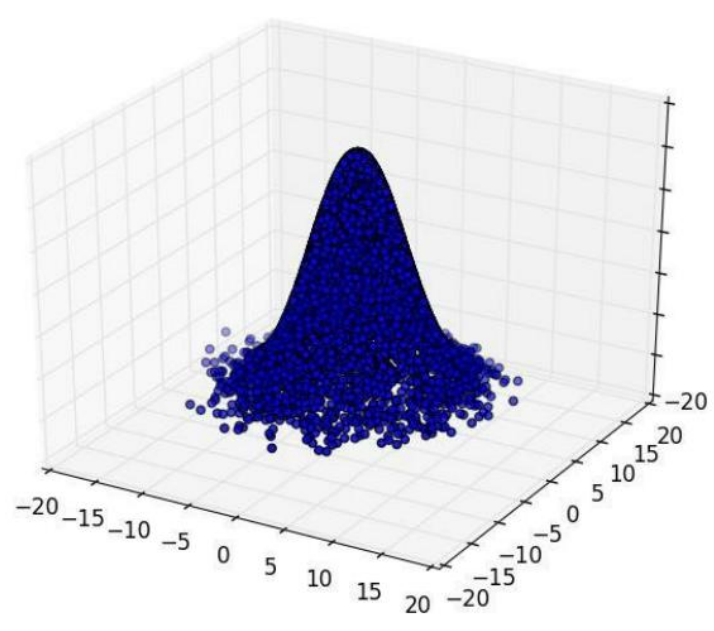

Figure 4. Schematic diagram of probability distribution density of underwater threat

\section{CONCLUSION}

It is a necessary requirement for the future naval warfare to develop submarine cooperative detection control, submarine cooperative attack and submarine command. Intelligent search and identification is a key problem to be resolved. The experimental results show that the MC algorithm is effective to get the underwater threat distribution low and underwater threat probability distribution density function. The shape, region size and the threat probability distribution in the region are more intuitively displayed compared to the previous algorithms. And it has a high military value [5] to meet the need of the future naval warfare.

\section{REFERENCES}

[1] F.X. LU, R. LI, L.J. TIAN, AEIT 2011.In Chinese.

[2] W.B. HUANG, X.Y. YU, B.C. LI, Fire Control\& Command Control. 38(2013), 169-176

[3] H. DU, X.Y. YU, C.M. SHI, 35(2015), Ship Electronic Engineering. 35(2015), 28-29.In Chinese.

[4] Paradowski, L.R., IEEE Transactions on Aerospace and Electronic Systems. 33(1997), 126-133.

[5] C.P. ZHENG, Command Information System and Technology. 4(2013), 19-21.In Chinese.

[6] B.H. WANG, J.H. HUANG, Q.F. ZHANG, SHIP SCIENCE AND TECHNOLOGY. 28(2006), 75-77.In Chinese.

[7] Aokai. ZHANG, J. LING, Y.W. SUN, G.D. FU., Chinese Journal of Polymer Science.33(2015),721-731.

[8] P.F. GUO ,S.Z. YUN , Tsinghua Univ (Sci\& Tech). 47(2007), 14-18.In Chinese.

[9] Mohammad, S. Hashem, M. Reza, International Journal of Mining Science and Technology. 573-578.

[10] Z.N. GUAN, N.X. WANG, N. XU, Journal of Electronic Measurement and Instrument.29(2015),447-453.In Chinese. 